\title{
H-mode characterisation for dominant ECRH and comparison to dominant NBI or ICRF heating at ASDEX Upgrade
}

\author{
F. Sommer, J. Stober, C. Angioni, E. Fable, M. Bernert, Burckhart, V. Bobkov, \\ R. Fischer, C. Fuchs, R.M. McDermott, W. Suttrop, E. Viezzer \\ and the ASDEX Upgrade Team
}

\author{
Max-Planck-Institut für Plasmaphysik, EURATOM Association, Boltzmannstr. 2, 85748 Garching, Germany
}

\begin{abstract}
At ASDEX Upgrade the ECRH system has been upgraded to provide up to $4 \mathrm{MW}$ of heating power at $140 \mathrm{GHz}$ (or $2.2 \mathrm{MW}$ at $105 \mathrm{GHz}$ ). The power at $140 \mathrm{GHz}$ exceeds the minimum H-mode power threshold for typical high $I_{p}, B_{t}$ conditions by approximately a factor of two. The upgrade allows H-modes with dominant electron heating and significant electron-ion heat exchange to be studied, i.e. the situation expected in ITER. This paper reports on systematic studies varying the heating mix with NBI, ICRF and ECRH and its effect on pedestal parameters and core transport. The H-mode pedestal is hardly affected by the choice of heating mix, but the ion temperature in the plasma center is found to vary significantly. The ion channel dominates heat transport and ion temperature gradient modes (ITG) are found to be the most unstable microinstability in all the scenarios considered. $R / L_{T_{i}}$ at half radius reduces by a factor of two when $T_{e} / T_{i}$ increases from 0.9 to 1.5 . TGLF modelling of the electron and ion temperature and electron density profiles shows very good agreement with the experimental data when applying a realistic sawtooth model.
\end{abstract}

Keywords: ASDEX Ugrade, ECRH, ICRF, NBI, H-mode, GS2, TGLF, ASTRA, profiles, modelling PACS: 52.35.Py

\section{INTRODUCTION}

Plasmas in present day devices used for preparatory or fundamental studies are often heated dominantly via the ion channel and with strong cocurrent momentum input. This is due to the dominance of neutral beam injection (NBI [1]) systems, which are widely used because of their reliability and universal applicability. The different energy components of the neutral beam of $1 \times E_{\text {beam }}, 1 / 2 \times E_{\text {beam }}$ and $1 / 3 \times E_{\text {beam }}$ and the slowing down of the beam while penetrating the plasma leads to an ion heating of around $50 \%$ to $80 \%$ for typical plasma conditions at ASDEX Upgrade (AUG). For the experimental conditions considered in the paper, the energy distribution to electrons and ions by the ion cyclotron resonance frequency (ICRF [2]) heating is similar to the NBI for the usually used $\mathrm{H}^{+}$minority heating scheme, but without torque input [3]. Since the power of the electron cyclotron resonance heating (ECRH [4]) in non existent or moderate at most major devices this leads to a dominant ion heating of the plasma in the past or present day devices. This situation will change significantly in the future. On one side the use of ECRH and lower hybrid systems will increase, which only heat the electrons. On the other side the beam energy of the NBI is planned to be increased by a factor of 10 compared to today's systems and the heating by $\alpha$-particles $\left(E_{k i n}=3.5 \mathrm{MeV}\right)$ will start to play a dominant role. All theses factors will lead to dominant electron heating of the plasma and the question arises how this will influence the performance of the plasma.

Dominantly electron heated plasmas are generated so far mainly with decoupled electrons and ions, i.e. low densities, so that most projections towards future devices are inferred from dominant ion heated conditions. The potential danger of this approach is an over-estimation of the scaled fusion performance, which depends on $T_{i}$ and not $T_{e}$, since the ratio of $T_{e} / T_{i}$ may depend on the heating mix. Even worse, an increasing value of this temperature fraction is expected to increase the ITG dominated turbulent transport in the ion channel reducing $T_{i}$ further [5]. Additionally, the significantly reduced ratio between NBI-torque and plasma inertia for future fusion reactors may increase transport due to a reduction of rotational shear. The issue of the torque can be addressed by the additional usage of countercurrent injection [6]. Significant central electron heating is only achieved by using high-voltage beams (requiring negative ion sources as in ITER) or by wave heating. The most direct way is ECRH, for which $100 \%$ of the injected power is transferred to the electrons close to the respective cyclotron resonance. Some ICRF schemes exist which only 
heat electrons, but they imply typically worse RF power absorption than the standard $\mathrm{H}^{+}$minority scheme and are therefore tricky to establish in the full tungsten AUG. Usually these heating scenarios lead to an increase of the W concentration in the core in $\mathrm{H}$-mode plasmas with $\mathrm{H}$-factors close to or above unity. This happens due to an increased $\mathrm{W}$ influx from the ICRF antenna limiters and an inward transport of the impurities. The impurity peaking leads to increased plasma radiation and a degradation of the performance of the discharge. The replacement of NBI by wave heating also modifies torque and non ambipolar losses of fast ions at the plasma edge which might contribute to the radial electric field in the plasma edge and, therefore, influence the H-mode pedestal.

With the upgrade of the ECRH system at AUG to $4 M W$, typically twice the H-mode threshold power, it is now possible to access H-modes with dominant electron heating such that the effects of the heating mix can be studied. Kinetic profiles $\left(n_{e}, T_{e}, T_{i}, v_{\text {tor }}\right)$ are measured using the recently upgraded suite of diagnostics in the core and, with high spatial resolution, in the pedestal region (additionally $v_{p o l}$ and $E_{r}$ ). In the following, the upgrade of the ECRH system and the upgraded diagnostics are presented. The results are then described separately for a low power, high collisionality and a high power, low collisionality discharge. Following calculations of the radial electric field are presented. In the modelling chapter predictions on the most unstable linear modes using the gyrokinetic code GS2 [7] and core profile evolution using the trapped gyro-Landau fluid code TGLF [8] are shown for both discharges. Some correlations of the whole database of discharges are shown. First results are already published in [9, 10].

\section{UPGRADE OF THE ECRH SYSTEM AND DIAGNOSTICS}

The ECRH of AUG consists of two systems. The old system comprises 4 units which deliver $0.4 M W$ for $2 s$ at $140 \mathrm{GHz}$ to the plasma each [11] and can only be operated in pairs (effective 2 times $0.8 \mathrm{MW}$ for $2 s$ ). The new system delivers $4 M W$ for $10 s$ from four $1 M W$ units with independent timing capability. Details of the system can be found in [12]. The new system was designed with multi-frequency capabilities with a main frequency of $140 \mathrm{GHz}$ and a second frequency of $105 \mathrm{GHz}$ with $80 \%$ of the power. For this study only $140 \mathrm{GHz}$ are used.

Several diagnostic improvements allow the reduction of stochastic and systematic errors. Improvements in the coreCXRS hardware allow the measurement of reliable $T_{i}$-profiles within $12 \mathrm{~ms}$, which is only $10-20 \%$ of $\tau_{E}$ such that short blips of the $2.5 \mathrm{MW}$ NBI beam can be used, which hardly disturb the background plasma. These blips appear as spikes of the NBI power traces in figure 1. Additionally, edge CXRS systems allow the measurement of $T_{i}$, $v_{t o r}$ and $v_{\text {pol }}[13]$ very precisely in the H-mode pedestal using the same beam blips. This allows the determination of the radial electric field and its shear in the steep gradient region, which is supposed to be responsible for the improved confinement in H-mode. For the latter, which requires also radial shifts of the plasma to increase the radial resolution, the usage of beam blips increases uncertainties significantly. Accordingly the $E_{r}$ calculations discussed below are only feasible in phases with continuous operation of at least one $2.5 \mathrm{MW}$ beam, which allows meaningful comparison only at medium to high heating powers . With respect to the $T_{e}$ diagnostics, the temperature stabilisation of the ECEradiometers has been improved and the calibration of the Thomson scattering has been optimised. At the plasma edge the assumption on full optical thickness has been given up for the ECE-data interpretation. Instead, for each frequency, the full emission and reabsorption is calculated in the frame work of integrated data analysis [14], which is also used to determine consistent density profiles from Li-beam, Thomson scattering and FIR-interferometry [15].

\section{EXPERIMENTS: LOW POWER, HIGH $v^{*}$}

Typical time traces of the performed experiments are shown in figure 1 for a low power $1 M A, 2.5 T$ ELMy H-mode with a total heating power of $3.3 \mathrm{MW}$ and a high collisionality of $v^{*}=0.2\left(v^{*}=v_{e i} \cdot q \cdot R /\left(\varepsilon^{1.5} \cdot v_{t h . e}\right)\right)$. Within single shots the heating mix of NBI and ECRH was modified while keeping the total heating and recycling fluxes constant. The latter is achieved by feedback control of the plasma fuelling using an ion gauge as reference for the recycling flux in the divertor, $\Gamma_{0, d i v}$. Such discharges were run for varying levels of total heating power, $\Gamma_{0, d i v}$, plasma current and magnetic field. We use here $\Gamma_{0, d i v}$, since the line averaged density varies only moderately in AUG H-modes (typically $20 \%$ ) when gas fluxes are changed by an order of magnitude. The NBI heating scheme used one or more $60 \mathrm{keV}, 2.5 \mathrm{MW}$ beams in either continuous or diagnostic blip operation in combination with 2 beams running at a reduced power of $0.8 \mathrm{MW}(52 \mathrm{keV})$. With this combination several steps of the total NBI power of $\approx 0.8 \mathrm{MW}$ are possible, thus enabling an even power exchange with the ECRH. Additionally discharges where performed, in which the power from ICRF $\mathrm{H}^{+}$minority heating was exchanged stepwise by ECRH power in the same manner. We note here that the boron coated limiters of the antennas significantly reduced the W influx during ICRF operation [16]. ECRH 

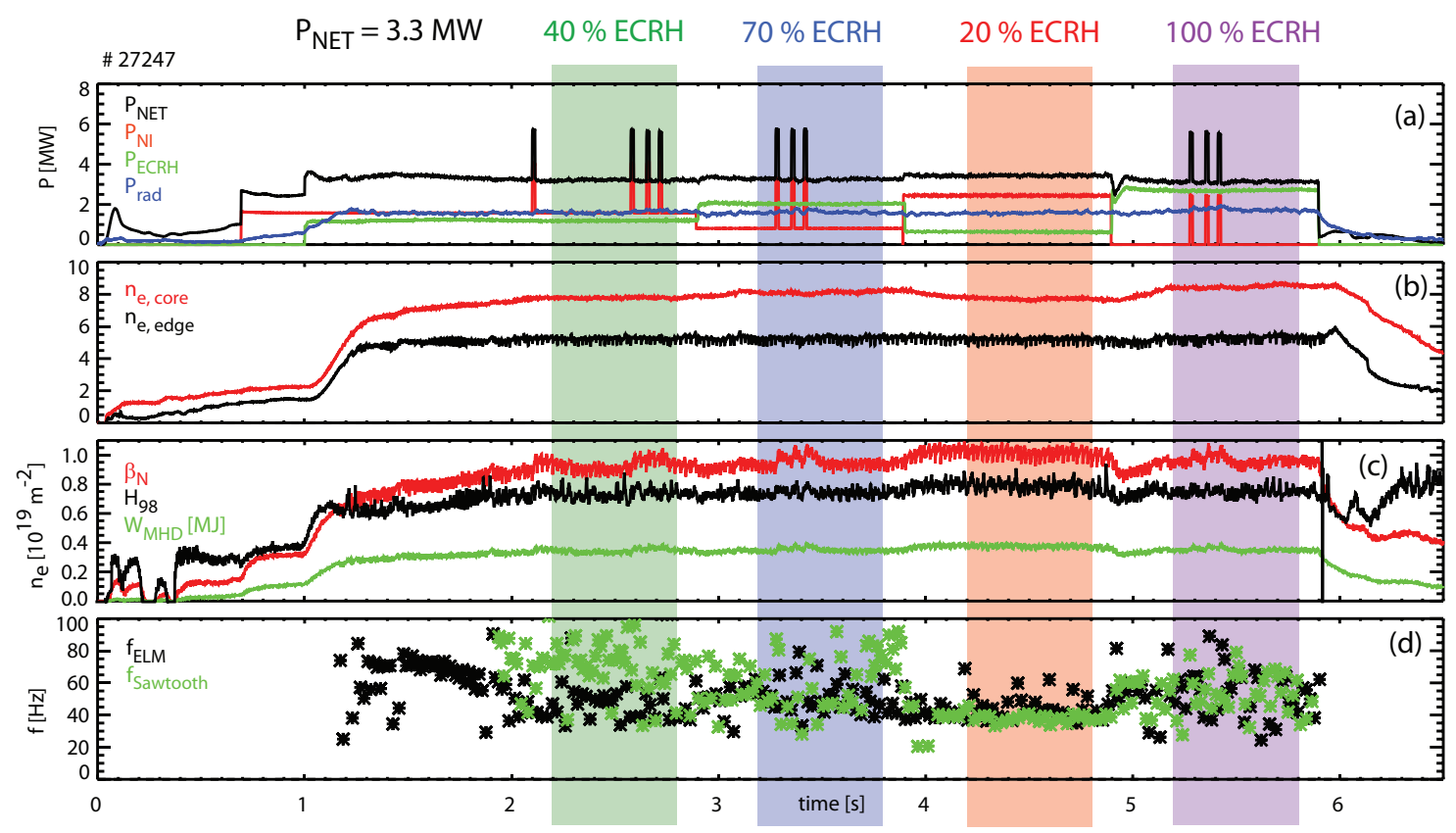

FIGURE 1. Plot (a): variation of the heating mix of ERCH (green) and NBI (red) with constant total heating power (black). The spikes in the NBI time trace correspond to diagnostic beam blips, the radiated power is shown in blue. Plot (b): line averaged densities for two lines of sight tangential to flux surfaces at $\rho_{\text {tor }}=0.1$ (core, red) and 0.8 (edge, black). Plot (c): stored energy (red) and derived quantities: $\beta_{N}$ in red and $H_{98}$ in black. Plot (d): ELM and sawtooth frequencies. The length of the different heating phases of $1 s$ corresponds to $\approx 10 \tau_{E}\left(I_{p}=1.0 \mathrm{MA}, B_{t}=-2.5 T, q_{95}=4.0\right)$. Marked with coloured bars are the steady state phases used for further analysis.

deposition was typically inside the $q=1$ surface to minimises $\mathrm{W}$ accumulation (empirical observation). The sawtooth (ST) crashes could not be stabilised by electron cyclotron current drive for the entire discharge. Sawtooth crashes and ELMs generally tend to lock and the degree of locking varies non monotonously with the fraction of ECRH as can be seen from figure 1 . The global plasma parameters do vary, but only within $10 \%$ as the ECRH contribution is varied from $20 \%$ to $100 \%$.
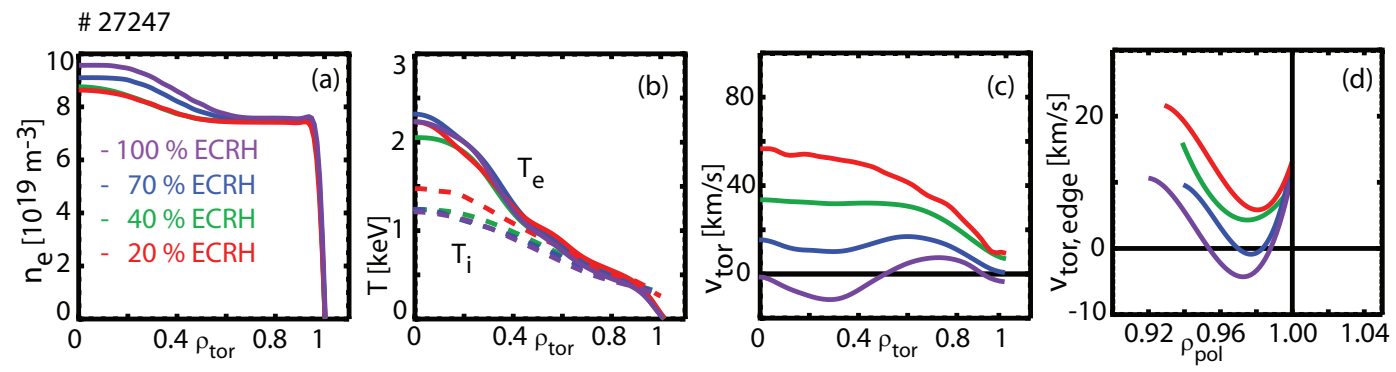

FIGURE 2. Plot (a-c): core profiles of electron density, electron and ion temperature and toroidal rotation for the low power discharge shown in figure 1. Plot (d): edge toroidal rotation.

In figure 2 the kinetic profiles from the intervals of different heating mix of the discharge presented in figure 1 are shown. In contrast to an often described pumpout due to ECRH, the electron density $n_{e}$ shows an increase of density peaking with ECRH fraction, which is typically observed in this collisionality range $[17,18] . T_{e}$ shows a $10 \%$ increase and $T_{i}$ a $15 \%$ decreases when going from $20 \%$ to $100 \%$ ECRH fraction. The toroidal rotation in the core and at the edge decreases with increasing ECRH fraction due to the reduced torque input by reduced NBI. The ECRH only case shows even counter current rotation which represents the intrinsic rotation of the plasma governed by the residual stress [19]. However the shape of $v_{t o r}$ at the edge is independent of heating mix. The pedestal values of $n_{e}, T_{e}$ and $T_{i}$ hardly vary as the heating mix is modified. 

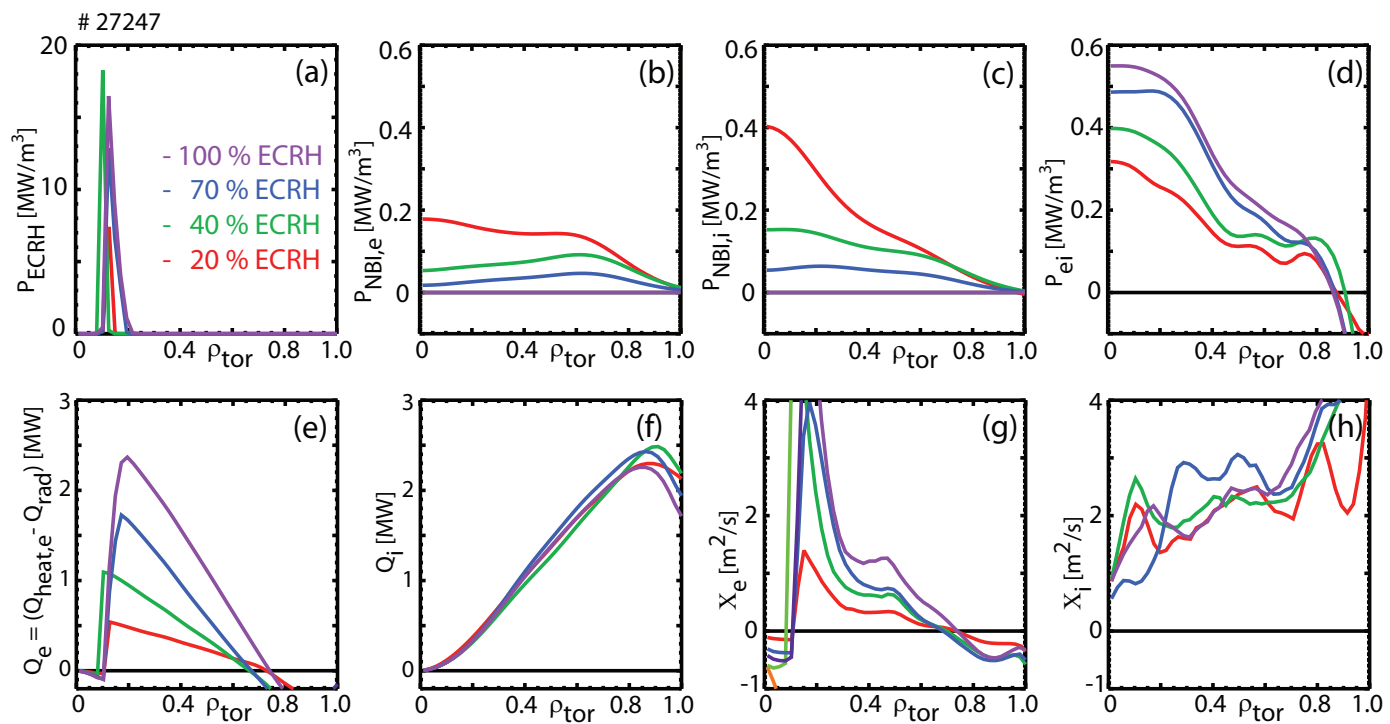

FIGURE 3. Transport analysis using ASTRA for the discharge shown in figure 1. The NBI heat deposition was calculated a priori using the experimental kinetic profiles and CLISTE equilibria with NUBEAM. Plot (a-d): heating power densities of ECRH $\left(P_{E C R H}\right)$, NBI to electrons $\left(P_{N B I, e}\right)$ and ions $\left(P_{N B I, i}\right)$ and heat exchange by Coulomb collisions from thermal electrons to thermal ions $\left(P_{e i}\right)$. Plot $(\mathrm{e}, \mathrm{f})$ : resulting flux-surface integrated heat-fluxes for both channels including ohmic heating and radiation losses in the electron channel. Plot (g,h): heat conductivities for both channels.

Figure 3 shows the results of a transport analysis using the transport analysis code ASTRA [20]. The NBI deposition is determined with the Monte Carlo code NUBEAM [21], the ECRH deposition with the beam tracing code TORBEAM [22] and the magnetic equilibrium with the equilibrium solver CLISTE [23]. The transport in the plasma center increases especially in the electron channel $\left(\chi_{e},(\mathrm{~g})\right)$ as ECRH is applied, which is expected for transport dominated by critical gradient lengths of the temperatures $R / L_{T}$ in case of central heat deposition in the electron channel. In the case shown here, $T_{e}$ and $T_{i}$-profiles adjust such that the central electron heating is transferred completely to the ion channel by local heat exchange due to Coulomb collisions $\left(P_{e i}\right)$ in the confinement region. Thus close to the pedestal top all the heat is flowing through the ion channel and $Q_{i}$ is independent of the heating mix (e,f). The nonphysical negative heat flux in the electron channel at the edge is fully within the experimental errors of the electron temperature. It can be avoided by an increase of $T_{e}$ by $5 \%$ or a reduction of $P_{r a d}$ by $50 \%$ in the power balance analysis. The figure indicates that $\chi_{e}$ and $\chi_{i}$ at half radius increases steadily as the fraction of ECRH is increased. Since these variations at half radius are close to the significance limit of the analysis, we do not attempt an interpretation here. The dominance of the ion heat channel indicates that the ITG-mode is most unstable in the confinement region.

\section{EXPERIMENTS: HIGH POWER, LOW $v^{*}$}

The collisionality of the discharge discussed above was rather high, i.e. $v^{*} \approx 0.2$ compared to 0.01 expected for ITER. In a next step the $100 \%$ ECRH phase was given up to allow total powers up to $8 \mathrm{MW}$. At this power level the maximum fraction of ECRH is limited to $45 \%$. Due to the centrally deposited ECRH and with $\approx 40 \%$ of electron heating by the beams, the heating power still went dominantly to the electrons. Figure 4 shows profiles of a discharge with a total heating power $\approx 2.5$ times higher compared to the discharge presented in figures $1,2,3$. The collisionality $v^{*}$ was reduced by roughly a factor of 4 to 0.05 . The electron density shows a large peaking but no variation with heating mix (plot (a)) which is in line with the transient region from ITG to trapped electron mode (TEM) dominated plasma (see GS2 calculations below). Also the steady state $T_{e}$ is unchanged, however $T_{i}$ decreases by $40 \%$ when increasing the ECRH fraction. The heat exchange between the channels (plot (d)) increases with heating mix and is significant smaller than the heating contributions but sufficient large to result in an ion heat flux which is independent of the heating mix. In contrast to the low power case, the heat flux in the electron channel is not transferred completely but has a finite value at the edge. $\chi_{e}$ and $\chi_{i}$ increase with increasing ECRH fraction as in the low power discharge. 

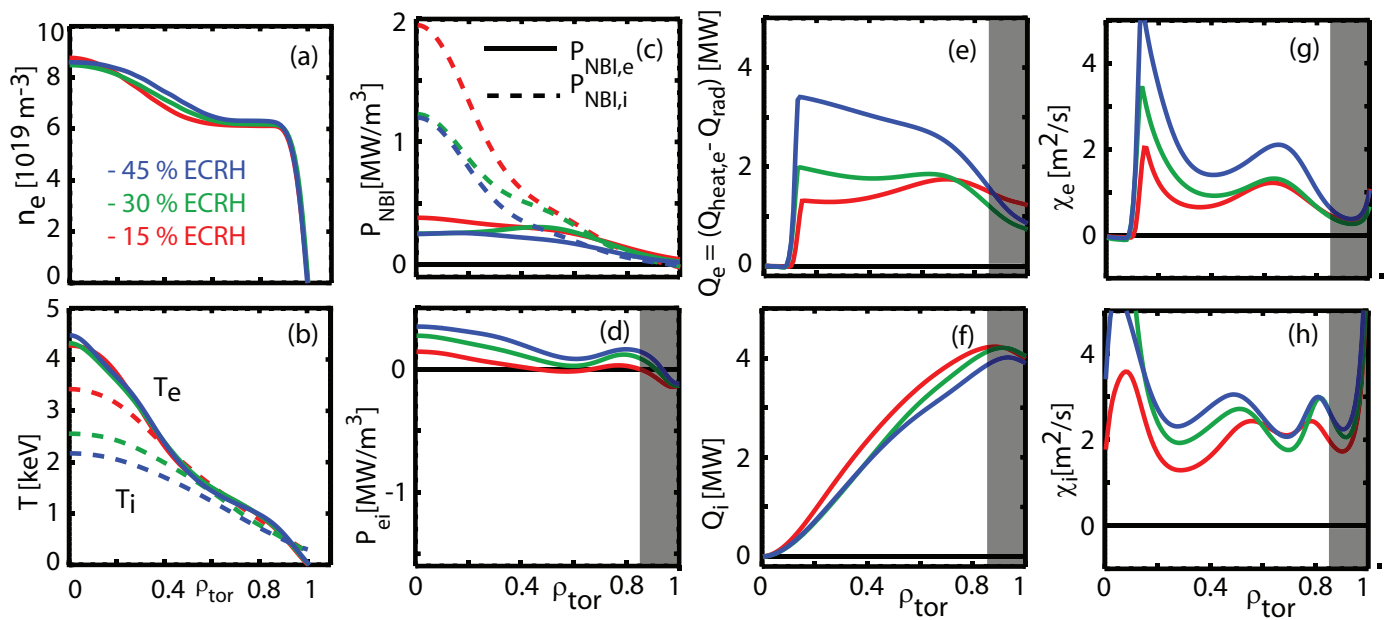

FIGURE 4. Plot (a,b): profiles of $n_{e}, T_{e}$ and $T_{i}$ for a discharge with a high total heating power of $8 M W, 2.5$ times the value of the discharge presented in figures 1,2,3 but otherwise identical parameters. Plot (c,d): heating power densities of NBI to electrons $\left(P_{N B I, e}\right)$ and ions $\left(P_{N B I, i}\right)$ and heat exchange by Coulomb collisions from thermal electrons to thermal ions $\left(P_{e i}\right)$. Plot (e-h): fluxsurface integrated heat fluxes and heat conductivities for both channels.

\section{RADIAL ELECTRIC FIELD}
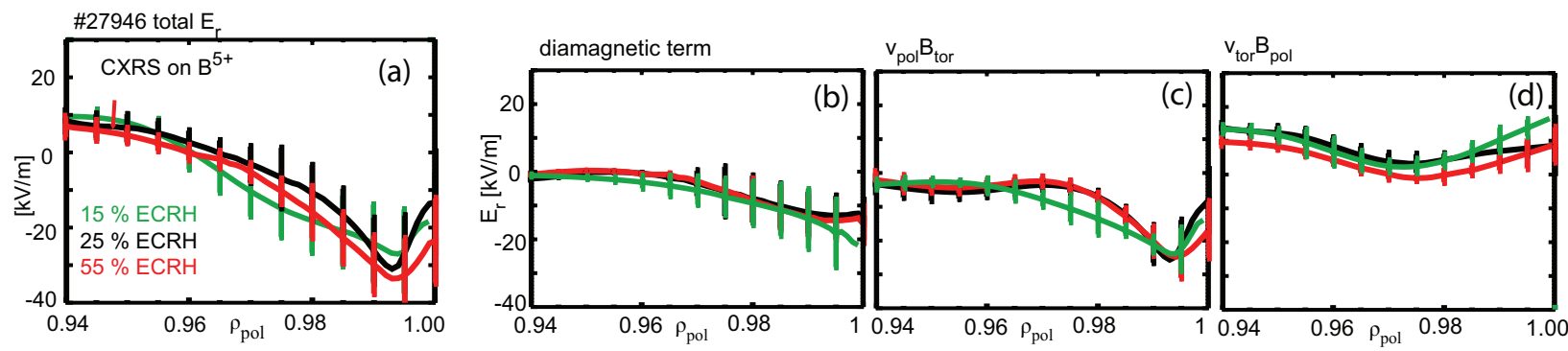

FIGURE 5. Plot (a): edge profiles of the total radial electric field at the edge for a discharge with a total heating power of $5.5 \mathrm{MW}$ (55\% ECRH: red, $25 \%$ ECRH: black, $15 \%$ ECRH: green), otherwise similar parameters as both discharges presented so far. Plot (b-d): three independent components of $E_{r}$ originating from the pressure gradient (diamagnetic term) and the poloidal and toroidal rotation, all inferred from the respective impurity ion species, here $B^{5+}$.

As discussed above a profile of the radial electric field can only be obtained using constant power of the NBI instead of beam blips. In figure $5 E_{r}$-profiles for three different mixtures of ECRH and NBI in discharge \# 27946 are shown together with the contributions due to the pressure profile and due to the poloidal and toroidal rotations. All three components are inferred from $\mathrm{B}^{5+}$. The $E_{r}$ profiles at the edge are indistinguishable. The different toroidal rotation only leads to a small variation within the error bars. In the case analysed here, the variation of the heating mix hardly varies the ratio of the power flowing through the ion and electron channel at the pedestal top (see above). Therefore we cannot make a statement on the effect of the variation of the heat fluxes in the different channels at the pedestal top. Here, the major effect of the changeover from NBI to ECRH is a reduction of the torque and the fast ion losses at the plasma edge, which both are proportional to the NBI power. We conclude that both parameters do not affect $E_{r}$ in the steep gradient zone within the error bars. These results fit nicely to the analysis of $E_{r}$ for H-modes under various conditions in [24], which shows that generally $E_{r}$ fits well to the diamagnetic term of the main ions as expected to zeroth order from neoclassical theory. In other words, rotation of the main ions is found to be a minor contribution to $E_{r}$ in the edge. 

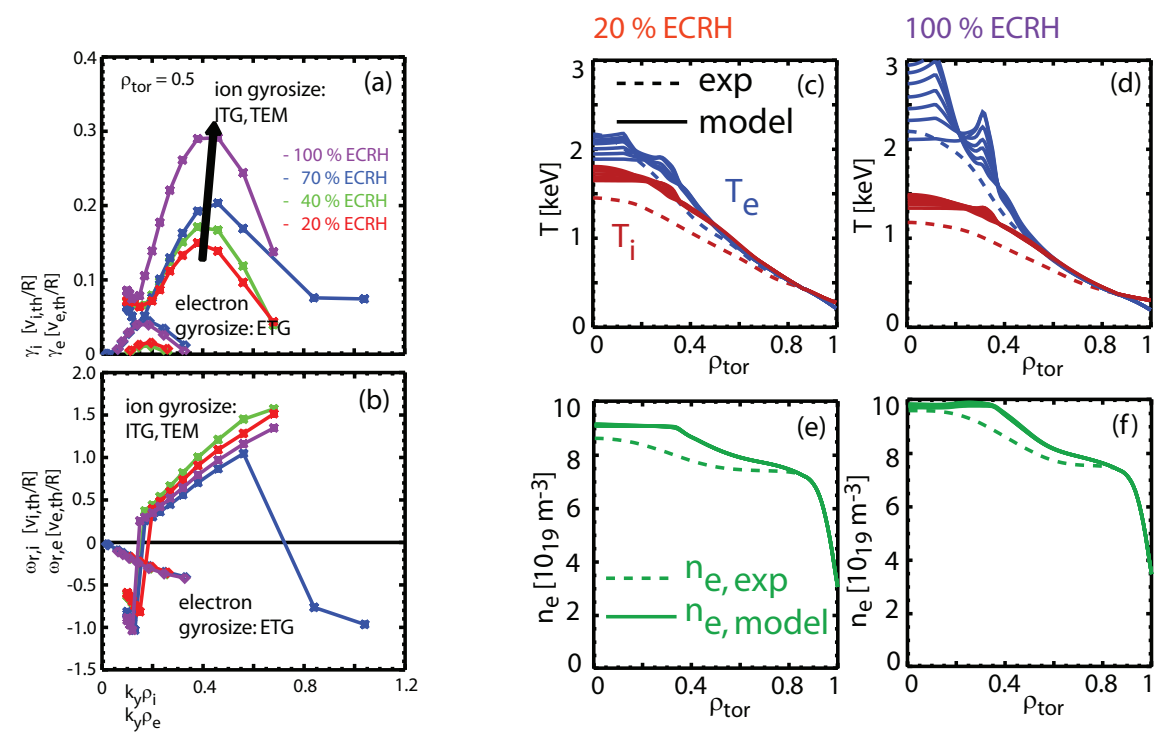

FIGURE 6. Plot (a,b): growth rates and frequencies of microinstabilities as determined with GS2 for the discharge shown in figure $1,2,3$ at $\rho_{\text {tor }}=0.5$. The growth rates and frequencies are normalised to $v_{t h} / R$ and the mode numbers to $k_{y} \rho$ of the respective species, i.e. ETG values are reduced by $\sqrt{m_{D} / m_{e}}$. Positive frequencies correspond to a rotation in the ion diamagnetic-drift direction. Plot (c-f) show the simultaneous TGLF modelling of the temperature and density profiles for the two extreme phases of this discharge i.e. $20 \%$ ECRH $(\mathrm{c}, \mathrm{e})$ and $100 \% \mathrm{ECRH}(\mathrm{d}, \mathrm{f})$. The different modelled profiles (solid lines) correspond to different times during one sawtooth cycle, while the experimental values are shown as dashed lines.

\section{MODELLING}

Analysis with the gyrofluid code GS2 of the low power high $v^{*}$ discharge \# 27247 shows a clear excess of the normalised growth rates of the ITG/TEM branch compared to those of the electron temperature gradient (ETG) branch (figure 6 (a)). Additionally the mode frequencies are positive which implies a rotation in the ion diamagnetic-drift direction and identifies the mode as ITG-like (plot (b)). With an increase of the ECRH fraction no fundamental change of the dominant microinstability can be found. However a drastic increase of the growthrate and an accompanying increase of the quasi linear cross phase with ECRH fraction is evident and indicates an increased heat flux in the ion channel. This is in line with the $\chi_{i}$ increase seen in the power balance analysis.

In order to test the quantitative predictability of the core transport we use the trapped gyro-Landau fluid code TGLF inside ASTRA, which is a first principle based model without any tuning parameters. The experimental values of $T_{e}$ and $T_{i}$ at $\rho_{t o r}=0.85$ are used as boundary condition and experimentally rotation profiles are prescribed for the entire radius. For the understanding of the latter significant progress has recently been made on AUG as reported in [25]. Figure 6 (c-f) shows the result for the two extreme cases of $20 \%$ and $100 \%$ ECRH for the combined temperature and density modelling. A realistic Kadomtsev-Porcelli sawtooth model has to be applied, which is crucial to prevent $T_{e}$ and $T_{i}$ peaking. The nonphysical peaks of the temperatures around $\rho_{t o r}=0.4$ are caused by the coupling of TGLF and the sawtooth model. The different profiles of the modelling correspond to several time points during one sawtooth cycle. The modelling shows very good agreement with the experiment. The profiles of the electron temperature, the increasing $T_{e} / T_{i}$ with ECRH fraction and the sawtooth crashes including their magnitude, radial extension and energy expulsion are reproduced very well. The ion temperature is in general a little overestimated in the high $v^{*}$ discharge. The shape of the experimental density profile and the increased peaking with increased ECRH fraction is recovered by the modelling very well. The modelling of the kinetic profiles does not show any sensitivity on the starting profiles realised by an exchange of the two cases. The sensitivity on the boundary conditions was analysed by a multiplication of $T_{e}$ and $T_{i}$ by 1.2 and 1.4 independently. The influence on the modelled profiles is restricted to $\rho_{t o r}>0.7$ due to a fast equipartition of $T_{e}$ and $T_{i}$. The influence of the ExB shearing on the profiles is less than $5 \%$, which is in line with the observation of very similar profiles with NBI and ICRF heating which have very different toroidal rotation profiles.

The same modelling has been performed for the high power, low $v^{*}$ discharge presented in figure 4 . Figure 7 (a,b) shows the dominance of the ITG microinstability also for this case. However with increasing ECRH fraction the mode 

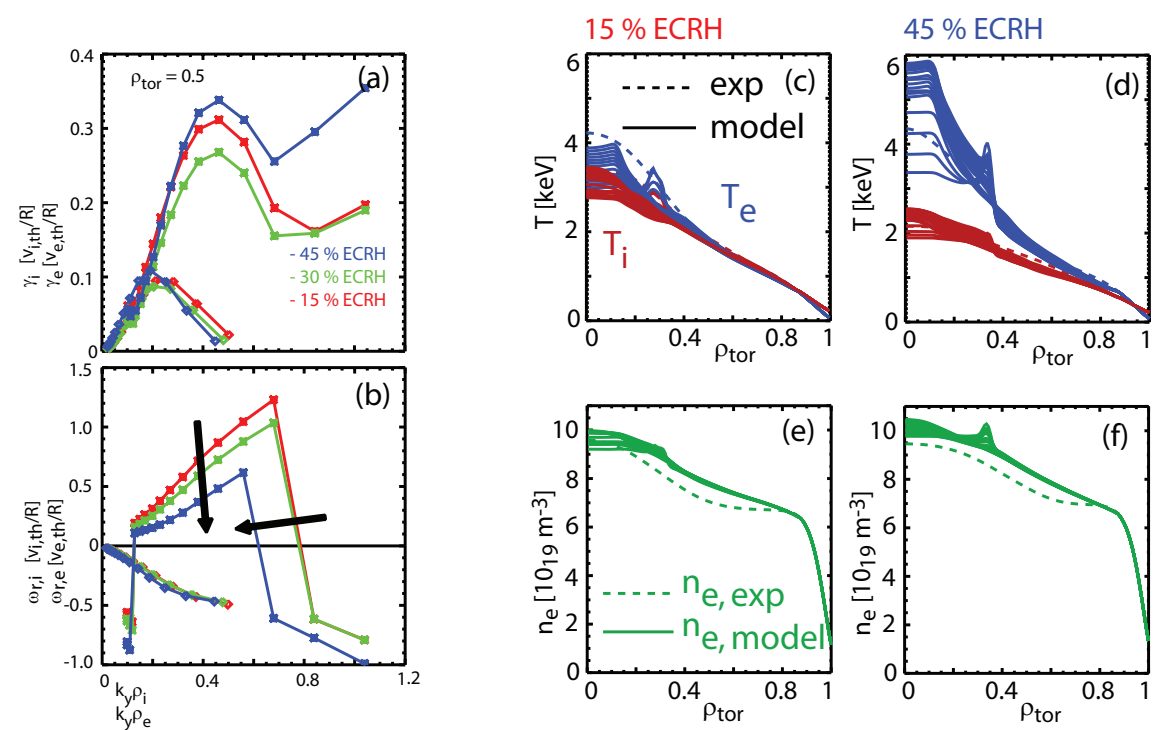

FIGURE 7. GS2 calculations and TGLF modelling as shown in figure 6 for the high power discharge presented in figure 4.

Plot $(a, b)$ : normalised growth rates and frequencies for the most unstable modes. Reduction of the mode frequency and approach of the jump from positive to negative frequencies towards the peak of the growthrate indicate increasing influence of TEM.

Plot (c-f): modelled temperatures and densities with TGLF for the two extreme phases of this discharge i.e. $15 \%$ ECRH (c,e) and $45 \%$ ECRH (d,f) applying a realistic sawtooth model.

frequency is reduced and the jump from positive to negative frequencies approaches the region of the most unstable mode. Both observations indicate a growing importance of the TEM and show the approach to the transition from ITG to TEM dominated turbulent transport. However all analysed phases are still ITG dominated and the contribution of TEMs to heat transport is negligible from linear gyrokinetic calculations. The modelling of the kinetic profiles of this discharge agrees very well with the experimental data, however the modelled electron temperatures show some discrepancy to the experimental profiles (figure 7 (c-f)). On the other side $T_{i}, n_{e}$, the sawtooth crashes, the increasing $T_{e} / T_{i}$ and the constant peaking of $n_{e}$ with increasing ECRH fraction are reproduced very well.

\section{CORRELATIONS}
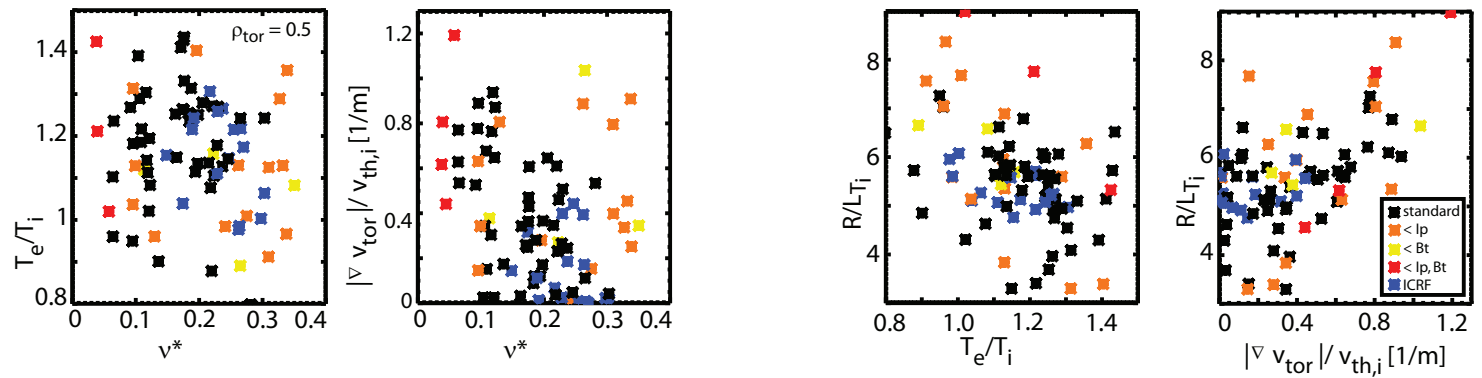

FIGURE 8. Plot (a,b): dependence of $T_{e} / T_{i}$ and $\left|\nabla v_{t o r}\right| / v_{t h, i}$ with $v^{*}$ abundant at $\rho_{t o r}=0.5$ for the overall database using mixtures of NBI, ECRH and ICRF (blue). Black and blue symbols: $q_{95}=4.0, I_{p}=1 M A$, red symbols: $q_{95}=4.0, I_{p}=0.75 M A$, yellow symbols: $q_{95}=3.0, I_{p}=1 M A$, orange symbols: $q_{95}=5.3, I_{p}=0.75 M A$. Plot (c,d): dependence of $R / L_{T_{i}}$ of $T_{e} / T_{i}$ and $\left|\nabla v_{\text {tor }}\right| / v_{t h, i}$. Gradients are taken from spline-fitted data.

Additionally to the two presented discharges, the data base was further extended using combinations of NBI, ECRH and ICRF at different total heating powers, lower plasma currents $(0.75 \mathrm{MA})$ and electron densities. Variations of the magnetic field from $2.5 T$ to $1.8 T$ were achieved using either X2 or X3 ECRH at $140 \mathrm{GHz}$. In figure 8 some of the dependencies of this data base are shown at $\rho_{t o r}=0.5$. The ratio $T_{e} / T_{i}$ and $\left|\nabla v_{t o r}\right| / v_{t h, i}$ do not correlate with $v^{*}$ due to 
the variation of the heating mix and total power. Plots $(a, b)$ show the achieved parameter space. With respect to ITG dominated transport the gradient length of $T_{i}$ is expected to depend on $T_{e} / T_{i}$, but also on the toroidal velocity, which interacts by destabilisation of the ITG via the parallel shear $\nabla v_{\|}$but also by stabilisation of the ITG via $E \times B$ shear. Consequently the data set is also plotted showing the dependence of $R / L_{T i}$ on $T_{e} / T_{i}$ and $\left|\nabla v_{t o r}\right| / v_{t h, i}$. The expected trend is indeed found for both parameters although for $\left|\nabla v_{t o r}\right| / v_{t h, i}<0.4$ no significant effect of the rotational shear on $R / L_{T i}$ is visible. These data nicely link to an older study on the branch $0.7 \leq T_{e} / T_{i} \leq 1.05$ at AUG still with the uncoated $\mathrm{C}$-tiles as plasma facing components allowing significantly lower densities [26]. Therein a combined effect of $T_{e} / T_{i}$ and $R \nabla v_{t o r} / v_{t h, i}$ could be identified to describe the $R / L_{T i}$ quantitatively well, stressing the importance of $E \times B$-shear stabilisation of the ITG.

\section{SUMMARY, CONCLUSIONS AND OUTLOOK}

Using the upgraded ECRH system of AUG, $\mathrm{H}$-mode operation with dominant electron heating and significant heat exchange between electron and ion channel has been studied for a wide range of $v^{*}$ ranging from 2 to 20 times the ITER value. For constant total power, recycling fluxes and plasma current the pedestal profiles and the radial electric field in the steep gradient region are not affected by the heating mix of ECRH, ICRF and NBI. In contrast, core transport is significantly modified and $T_{i}$ decreases with increasing ECRH fraction in steady state. Over the whole range of collisionalities ion transport is dominant and the ITG is the most unstable mode with an approach towards TEM at very low collisionalities and high ECRH fraction. Theoretically it is expected that the onset of the ITG depends crucially on $T_{e} / T_{i}$. Experimentally it is found indeed that for $0.9<T_{e} / T_{i}<1.6, R / L_{T i}$ drops from $\geq 8$ to $\leq 5$ at $\rho_{\text {tor }}=0.5$. The proposed mechanism for the performance loss with ECRH fraction is an increase of $T_{e}$ with ECRH. The resulting increase of $T_{e} / T_{i}$ increases transport in the ion channel (ITG) so that $T_{i}$ is reduced, which also drags down $T_{e}$. Experimental $n_{e}, T_{e}$ and $T_{i}$ profiles can be modelled quantitatively very well with TGLF, using the experimentally determined rotation profiles and a realistic sawtooth model. The database still shows a large scatter of $R / L_{T i}$ as function of $T_{e} / T_{i}$ and $\left|\nabla v_{t o r}\right| / v_{t h, i}$. The analysis of cross correlations and error bars and the dependence on machine conditions and discharge evolution ( $q$-profile) is ongoing. Reliable predictions of $T_{e} / T_{i}$ are crucial for ITER given the dominance of the electron heating.

\section{REFERENCES}

1. STREIBL, B. et al., Fusion science and technology, 44(3):578-592, 2003.

2. FAUGEL, H. et al., Fusion engineering and design, 74(1-4):319-324, 2005.

3. BILATO, R. et al., Nucl. Fusion, 51(10):103034, 2011.

4. STOBER, J. et al., EPJ Web of Conferences, 32:02011, 2012.

5. GUO, S. C. et al., Physics of Fluids, 5(2):520-533, 1993.

6. SCOVILLE, J. T., Fusion Sci. Technol., 52:398-403, 2007.

7. KOTSCHENREUTHER, M. et al., Comput. Phys. Commun., 88:128, 1995.

8. STAEBLER, G. M. et al., Phys. Plasmas, 14:055909, 2007.

9. SOMMER, F. et al., Nucl. Fusion, 52:114018, 2012.

10. STOBER, J. et al., Proceedings, IAEA-CN-197:EX/1-4, 2012.

11. LEUTERER, F. et al., Fusion Science and Technology, 55(1):31-44, 2009.

12. WAGNER, D. et al., Nucl. Fusion, 48(5):054006, 2008.

13. VIEZZER, E. et al., Review of Scientific Instruments, 83(10):103501, 2012.

14. RATHGEBER, S. K. et al., Plasma Physics and Controlled Fusion, 55(2):025004, 2013.

15. FISCHER, R. et al., Fusion Science and Technology, 58(2):675-684, 2010.

16. BOBKOV, V. et al., Proceedings, IAEA-CN-197:EX/P5-19, 2012.

17. ANGIONI, C. et al., Nucl. Fusion, 51(2):023006, 2011.

18. FABLE, E. et al., Plasma Physics and Controlled Fusion, 52(1):015007, 2010.

19. ANGIONI, C. et al., Phys. Rev. Lett., 107:215003, 2011.

20. PEREVERZEV, G. et al., IPP Technical Report, 5/98, 2002.

21. PANKIN, A. et al., Comput. Phys. Commun., 159:157-184, 2004.

22. POLI, E. et al., Physics of Plasmas, 6(1):5-11, 1999.

23. CARTHY, P. et al., Plasma Physics and Controlled Fusion, 54(1):015010, 2012.

24. VIEZZER, E. et al., Nucl. Fusion, 53(5):053005, 2013.

25. MCDERMOTT, R. et al., Proceedings, IAEA-CN-197:EX/2-1, 2012.

26. MANINI, A. et al., Nucl. Fusion, 46(12):1047-1053, 2006. 